\title{
Underground Pipeline Response to Earthquake-Induced Ground Deformation
}

\author{
T.D. O'Rourke ${ }^{1}$, J. K. Jung ${ }^{2}$, and C. Argyrou ${ }^{3}$
}

\begin{abstract}
The principal causes of earthquake-induced ground deformation are identified and their interaction with underground infrastructure, primarily pipelines and conduits, is described. The coupled forces normal and parallel to underground pipelines arising from earthquake-induced ground movement are evaluated, including a review of measured stresses on pipe surfaces during large-scale testing, evaluation of frictional forces related to soil-pipe interaction, and the resolution of interaction forces along and across pipelines. Methods for characterizing soil reaction to pipe lateral and vertical movements are presented. The maximum downward pipe force is only about one-third the maximum force determined with conventional bearing capacity equations, thus requiring changes in current analytical and design practice. The analytical results for pipeline response to strike-slip and normal fault rupture are shown to compare favorably with the results of both large-scale and centrifuge tests of soil-pipeline interaction simulating these types of severe ground deformation.
\end{abstract}

\section{Introduction}

Earthquake-induced ground deformation is a major concern for underground infrastructure in areas vulnerable to seismic risk. It is also representative of extreme conditions of soil-structure interaction that accompany floods, hurricanes, landslides, large soil movements caused by tunneling and deep excavations, and subsidence resulting from dewatering and/or withdrawal of minerals and fluids during mining and oil production. Hence, pipeline performance during earthquakes provides a framework for the analysis and design of underground infrastructure that is resilient to a variety of natural and construction-related hazards.

This paper focuses on methods for analyzing underground pipeline and conduit response to large permanent ground deformation caused by earthquakes. It begins with a review of the sources of earthquake-induced soil movement and shows how these movements are converted into soil-pipe reactions normal and parallel to the longitudinal axis of the pipeline. Two-dimensional (2D) finite element (FE) analytical approaches are described, and improved methods are presented for 2D modeling of the coupled forces normal and parallel to underground pipelines during large permanent ground displacements. Methods for characterizing soil reaction to pipe lateral and vertical movements are presented with reference to large-scale tests involving pipe lateral and uplift movement in dry and partially saturated sand as well as plane strain FE soil and pipe continuum models. The analytical results for vertical downward movement of pipe in soil are

\footnotetext{
${ }^{1}$ Professor, Sch. of Civil \& Envr. Engr., Cornell University, Ithaca, NY, USA tdo1@ @ornell.edu

${ }^{2}$ Assistant Professor, Civil \& Envr. Engr., Youngstown State Univ., Youngstown, OH, USA jai.k.jung@gmail.com

${ }^{3}$ Graduate Research Assistant, Sch. of Civil \& Envr. Engr., Cornell University, Ithaca, NY ca353@ cornell.edu
} 
presented. Analytical results for pipeline response to strike-slip and normal fault rupture are shown to compare favorably with the results of both large-scale and centrifuge tests of soilpipeline interaction simulating these types of ground deformation.

\section{Earthquake-Induced Ground Deformation}

As described previously (e.g., O'Rourke, 2010; O'Rourke and Liu, 2012), earthquakes cause transient ground deformation (TGD) and permanent ground deformation (PGD), both of which affect underground infrastructure. TGD is the dynamic response of the ground, and PGD is the irrecoverable movement that persists after shaking has stopped. It may involve pulses of strong motion that locally exceed soil shear and tensile capacity, causing surficial soil cracks and offsets. PGD frequently results in large movements, such as those associated with surface fault rupture, liquefaction-induced lateral spreading, and landslides.

The principal causes and types of TGD and PGD have been summarized by Bird et al. (2004) and are presented in Table 1. In aggregate, they represent the total seismic hazard affecting the performance of underground pipelines and conduits.

To illustrate soil-pipeline interaction, Figure 1a provides a plan view of an underground pipeline deformed by soil mass movement associated with a landslide or liquefaction-induced lateral spread. There is abrupt ground deformation at the margins of the slide/lateral spread where the pipeline is affected by movement oblique to its longitudinal axis. Insert A shows the direction of lateral soil movement, $d_{H}$, and horizontal soil reaction force/unit distance, $p_{H}$, at the left-hand side of the soil mass. Insert B shows the direction of lateral soil movement and soil reaction force/unit distance, $p_{H}$, at the center of the soil mass, where two-dimensional (2D) conditions control the horizontal force mobilized against the pipe. The plane strain conditions of soil-pipe interaction in Insert B are consistent with the large-scale tests and numerical simulations used to estimate the maximum horizontal soil reaction forces and force vs. displacement relationships for lateral soil-pipeline interaction (e.g., Trautmann and O'Rourke, 1985; Jung et al., 2013b). For 2D plane strain soil movement $p_{H}, d_{H}$, and frictional force/unit distance, $f_{N}$, are normal to the longitudinal axis of the pipeline.

As illustrated in Insert $\mathrm{B}, p_{H}$ under conditions of oblique soil movement is estimated from the large-scale test results and numerical simulations for 2D plane strain conditions in which lateral force vs. displacement relationships use the component of soil displacement, $d_{H}$, normal to the longitudinal axis of the pipeline (parallel to the normal frictional force, $f_{N}$ ). There is ample experimental evidence to justify this approach. Ha et al. (2008) report measured maximum horizontal soil forces under oblique soil movement in centrifuge tests on pipelines subjected to strike-slip fault displacement that compare favorably with those from large-scale 2D plane strain test results. Moreover, bending strains predicted from 2D finite element simulations of pipeline response to strike-slip fault movement compare very well with bending strains measured in large-scale and centrifuge tests of pipeline/strike-slip fault interaction (O'Rourke et al. 2008). The close agreement between experimental and simulated bending strains is noteworthy because bending strains are especially sensitive to $p_{H}$.

The frictional force, $f_{T}$, generated in granular soil during oblique soil movement is decomposed 
into its parallel component as $f_{P}=f_{T} \cos \beta$ to obtain the frictional force along the longitudinal axis of the pipeline. The frictional force component normal to the pipeline longitudinal axis is $f_{N}=$ $f_{T} \sin \beta$, which represents the friction dissipated as soil displaces around the pipe. Thus, the parallel and normal components of $f_{T}$, depend on the angle, $\beta$, which defines the obliquity of soil movement relative to the pipeline. Please note that $f_{T}$ is a vector, which is aligned in the same orientation as the oblique soil displacement, $d_{T}$.

The three-dimensional (3D) view in Figure $1 \mathrm{~b}$ shows a different orientation in which the pipeline is subjected to axial compression and tension at opposite sides of the moving soil mass. A pipeline crossing the margin of a slide/lateral spread is similar to one crossing an active strikeslip fault. The vector frictional force, $f_{T}$, is decomposed into normal and parallel components in the same way for both conditions of earthquake-induced soil deformation.

\section{Pipeline Frictional Forces}

For a pipeline at rest in cohesionless soil, it is well established (e.g., ASCE, 1984) that the maximum frictional force per unit distance along the pipeline longitudinal axis, $f_{\text {pmax }}$, is given by

$$
f_{p \max }=0.5\left(1+K_{0}\right) \bar{\gamma} H_{c} \pi D \tan \delta
$$

in which $H_{c}$ is the depth to pipe centerline, $\bar{\gamma}$ is the soil unit weight contributing to effective stress at the pipe centerline, $K_{0}$ is the coefficient of at-rest horizontal soil stress (generally $0.5 \leq$ $K_{0} \leq 1.0$ for pipe in backfilled trenches), $\delta$ is the angle of interface frictional resistance, and $D$ is the pipe outside diameter.

The force, $f_{\text {pmax }}$, is mobilized to resist axial pipeline deformation where at-rest conditions exist outside the area of major soil deformation, such as the landslide/lateral spread illustrated in Figure 1. When a pipeline or conduit is intersected by soil movement, the at-rest conditions are disrupted, and the pipeline is subjected to frictional force directly related to the distribution of stresses normal to the pipe surface and indirectly related to $p_{H}$, which is the soil reaction force normal to the pipeline longitudinal axis.

To understand how $p_{H}$ is related to the frictional force on the pipeline, pipe surface stress measurements with tactile pressure sensors are examined. As described by Palmer et al (2009), a tactile pressure sensor is an array of small sensing units, called sensels, embedded in a polymeric sheet or pad that measures the magnitude and distribution of stresses normal to the sheet surface. Measurements with these sensors during large-scale 2D tests of soil-pipe interaction under relative horizontal displacement, $\delta_{H}$, between pipe and soil (Palmer et al., 2009) were analyzed in accordance with Figure 2.

As a pipe displaces laterally in soil, approximately half the pipe circumference is drawn away from the soil, while the other half is pushed towards the soil mobilizing passive pressures. This observation has been validated by test measurements and described in previous publications (e.g., O'Rourke et al, 2008; Palmer et al, 2009). Thus, the pressure is exerted predominantly on half the pipe. In partially saturated sands, there is virtually no pressure on the back of the pipe, 
because suction holds the soil particles in place and a void typically opens behind the pipe. The tactile pressure sensor data confirm that, at least for pipe depth to diameter ratios, $\mathrm{H}_{\mathrm{c}} / \mathrm{D}$, of 3.5 to 7.5 , measured pressures were virtually zero from $\theta=\pi$ to $\theta=2 \pi$. This is consistent with the schematic of the pressure distribution shown in Figure 2.

If $p(\theta)$ denotes the soil stress normal to the pipe surface as a function of angular position around the circumference, $\theta$, and $\tau(\theta)=p(\theta) \tan \delta$ is the frictional shear stress, $p_{H}$ is obtained by integrating the horizontal components of $p(\theta)$ and $p(\theta) \tan \delta$ as follows

$$
p_{H}=\frac{D}{2}\left[\int_{0}^{2 \pi} p(\theta) \sin \theta d \theta+\tan \delta \int_{0}^{2 \pi} p(\theta)|\cos \theta| d \theta\right]
$$

The net horizontal force per unit length, $p_{H}$, can also be obtained from the experimental data using the following relation

$$
p_{H}=\sum_{j=1}^{J}\left(p_{m}\right)_{j} S_{j} \sin \theta_{j}+\tan \delta \sum_{j=1}^{J}\left(p_{m}\right)_{j} S_{j}\left|\cos \theta_{j}\right|
$$

in which $\left(p_{m}\right)_{j}$ is the measured pressure, $p_{m}$, at the $\mathrm{j}$-th pressure sensor node, $S_{j}$ is the arc length associated with the $\mathrm{j}$-th pressure sensor node $\left(S_{j}=2 \pi R / J\right), \theta_{j}$ is the angle defining the orientation of $\left(p_{m}\right)_{j}$, and $J$ is the total number of pressure sensor nodes around the pipe surface per unit length.

Normal stress measurements taken by Palmer et al. (2009) for soil-pipe interaction in dry sand were normalized with respect to the maximum normal stress, $p_{\max }$, and regressed with the sinusoidal curve fitting techniques described in Appendix A to obtain distributions of measured normalized stress, $p(\theta) / p_{\max }$. Two sets of measurements were regressed from tactile pressure sensors, denoted as Sensor B and Sensor A, located at the midpoint and a distance of $1 / 4$ the test pipeline length from its midpoint, respectively. The two datasets show slightly different normalized pressure distributions, and were selected to cover variations in pressure measured along the test pipeline. The regression analyses were performed on 24 and 12 different measurements at different levels of lateral soil movement for Sensor A and B, respectively. In each case, two erratic measurements were screened from the data at relatively low levels of lateral pipe movement before the maximum horizontal force was attained.

Figure $3 \mathrm{a}$ is a plot of the regression equation for normalized pressure measured by Sensor A regressed with respect to angular distance, $\theta$, from the pipe crown, as defined in the insert diagram. Also plotted in the figure are the normalized pressure distributions from finite element (FE) simulations using pipe shell and soil continuum elements performed by Jung et al. (2013b) for the same large-scale 2D test reported by Palmer et al. (2009). The FE results correspond to 20 and $30 \mathrm{~mm}$ of relative horizontal pipe displacement, which is consistent with the relative displacements pertaining to the experimental data. The analytical and experimental normalized pressures are in close agreement.

Figure $3 \mathrm{~b}$ provides an alternative way of visualizing the data in which the normalized pressure, 
transformed as an inverse sine function $\left(\sin ^{-1}\right)$ in accordance with Appendix A, is shown as a linear regression with respect to angular distance. The actual measurements are plotted in the figure. The linear regression involves rising and falling portions that are fit to the data by the least squares method.

For Sensor A, the normalized pressure distribution $\left(r^{2}=0.96\right)$ is given by

$$
\frac{p(\theta)}{p_{\max }}=0.526-0.454 \sin (2 \theta+1.344)
$$

The total normal force per unit distance, $p_{N}$, acting on the pipe can be calculated as the product of the maximum pressure and integration of the normalized stress distribution. Similarly, $p_{H}$, can be determined by means of Eqs. 2 and 3. Both numerical integration of tactile pressure sensor measurements and closed form integration of Eq. 4 are in excellent agreement, yielding

$$
\begin{aligned}
& p_{N}=\frac{D}{2} p_{\max } \int_{0}^{\pi} \frac{p(\theta)}{p_{\max }} d \theta=1.652 \frac{D}{2} p_{\max } \\
& p_{H}=\frac{D}{2} p_{\max }\left[\int_{0}^{\pi} \frac{p(\theta)}{p_{\max }} \sin \theta d \theta+\int_{0}^{\pi} \frac{p(\theta)}{p_{\max }} \tan \delta|\cos \theta| d \theta\right]=\frac{D}{2} p_{\max }(1.346+0.757 \tan \delta)
\end{aligned}
$$

The total frictional force, $f_{T}$, is the product of the normal force acting on the pipe and $\tan \delta$ of the pipe surface, as follows

$$
f_{T}=p_{N} \tan \delta=1.652 \frac{D}{2} p_{\max } \tan \delta
$$

The apparent frictional force per unit pipe length, $f_{A}$, can be defined as

$$
f_{A}=\mathrm{p}_{H} \tan \delta=\frac{D}{2} p_{\max }(1.346+0.757 \tan \delta) \tan \delta
$$

It is convenient to estimate the longitudinal friction force during numerical simulation as the product of the force normal to the longitudinal pipeline axis and $\tan \delta$. Therefore, the ratio, $f_{T} / f_{A}$, is a correction factor that can be applied to the apparent friction, $f_{A}$, to reflect the total friction acting on the pipe. Combining Eqs. 7 and 8 results in

$$
\frac{f_{T}}{f_{A}}=\frac{1.652}{1.346+0.757 \tan \delta}
$$

Figure 4a shows $f_{T} / f_{A}$ relative to tan $\delta$ for both Sensor A and B measurements. Figure 4b provides a plot of the Sensor A and B measurements compared with the calculations of $f_{T} / f_{A}$ vs. $\tan \delta$ from finite element (FE) simulations of lateral, upward, and downward pipe movement that are described under forthcoming sections of this paper. As shown in Figure 4b, the experimental and numerical data for different types of pipe movement are in close agreement. The $f_{T} / f_{A}$ vs. 
$\tan \delta$ relationship for Sensor A measurements is within $\pm 5 \%$ of the $f_{T} / f_{A}$ values for lateral and vertical pipe movements. Hence, the $f_{T} / f_{A}$ vs. $\tan \delta$ relationship for all orientations of pipe movement can be estimated with Eq. 9.

For polyethylene and polyvinyl chloride pipelines as well as pipelines with polyethylene or fusion epoxy coatings, $\tan \delta$ ranges between 0.3 and 0.6. As shown in Figure $4, f_{A}$ and $f_{T}$ are in close agreement for this range of $\tan \delta$, with variations up to $8 \%$. For concrete pipelines, pipelines with mortar coatings, and metallic pipelines without coatings $\tan \delta$ typically is between 0.7 and 1.0 so that $f_{A}$ is a conservative overestimate of $f_{T}$ by about $12-21 \%$.

Figure 4 indicates that $p_{H} \tan \delta$ can be used to estimate the longitudinal frictional force on a pipeline with little to no error when $0.4 \leq \tan \delta \leq 0.6$ and to provide a conservative estimate when $\tan \delta>0.6$. A reliable estimate of longitudinal frictional force, therefore, requires a coupled analysis in which $p_{H}$ and $\tan \delta$ are combined. It is often assumed that the at-rest conditions given by Eq. 1 apply along the entire pipeline for 2D pipeline response to soil movement. This assumption is not accurate in areas of major soil deformation, such as the landslide/lateral spread shown in Figure 1, and will result in an unconservative estimate of maximum axial pipe strain.

\section{Lateral and Uplift Soil-Pipe Interaction}

A comprehensive program of large-scale tests and FE modeling was undertaken to evaluate the maximum soil reaction force and force vs. displacement relationships for lateral and uplift movement of pipelines in dry and partially saturated sand (O'Rourke et al., 2008; O'Rourke, 2010). Reference should be made to Jung et al. (2013a and 2013b) for a detailed presentation of experimental and analytical findings. Only the salient features of the large-scale testing, FE modeling, and program results are provided in this paper.

Numerous large-scale tests were performed at the Large-Scale Lifelines Testing Laboratory at Cornell University, which was originally part of the George E. Brown Network for Earthquake Engineering Simulation (O'Rourke et al., 2008). The tests were performed in conjunction with tests using the 150 g-ton centrifuge at Rensselaer Polytechnic Institute (RPI). The test results were used to develop relationships between maximum soil reaction forces, sand density, and pipe depth/diameter as well as to validate FE models for lateral and uplift pipe movement in sand.

Figure 5 shows the split-basin testing facility at Cornell University that utilizes as much as 90 metric tons of partially saturated sand per test with as much as 1.2-1.8 $\mathrm{m}$ of strike-slip displacement. Large-scale tests were conducted with this facility on HDPE pipelines with nominal 250-mm and 400-mm-diameters. The pipelines were subjected to $1.22 \mathrm{~m}$ of strike-slip displacement at a crossing angle of $65^{\circ}$ with respect to vertical plane of ground rupture. The depth of cover was $900 \mathrm{~mm}$ to top of pipe in all tests. Each pipeline was instrumented with over 100 strain gages, many with the capability of measuring strains as high as $20 \%$. All tests were conducted with partially saturated sand, with suction of 4-5 kPa measured by tensiometers (O’Rourke et al. 2008) and strength and stiffness consistent with dense sand backfill. 
Soil-pipeline interaction for lateral and uplift pipe displacement in granular soil was evaluated by means of a two-dimensional, finite element (FE) continuum model with a Mohr-Coulomb (MC) yield surface for peak strength, a strain softening relationship tied to critical void ratio, and an equivalent modulus that is consistent with soil deformation at maximum lateral and uplift resistance (Jung et al., 2013a and 2013b). A systematic comparison of FE results with multiple full-scale test measurements show excellent agreement both with respect to maximum force and force-displacement relationships, including post-peak performance.

Figs $6 \mathrm{a}$ and $6 \mathrm{~b}$ and Figs. $6 \mathrm{c}$ and $6 \mathrm{~d}$ compare representative analytical and large-scale test results for lateral and uplift pipe-soil interaction, respectively. The figure shows the dimensionless force, $\mathrm{F} /\left(\mathrm{H}_{\mathrm{c}} \bar{\gamma} \mathrm{DL}\right)$ vs dimensionless displacement, $\mathrm{Y} / \mathrm{D}$, for which $\mathrm{F}$ is the pipe-soil reaction force, $\bar{\gamma}=$ soil unit weight contributing to effective stress at the pipe centerline, $\mathrm{H}_{\mathrm{c}}=$ depth to pipe center, $\mathrm{D}=$ outside pipe diameter, $\mathrm{L}=$ pipe length, and $\mathrm{Y}=$ relative soil-pipe displacement. Please note that the experimental and analytical test results reported for dry sand in this work pertain to effective stress conditions without suction generated in unsaturated sand. The largescale test and analytical results compare favorably both for dry and partially saturated sand. Of particular interest is the simulation of strain softening behavior for lateral pipe movement in partially saturated sand with $\mathrm{H}_{\mathrm{c}} / \mathrm{D}=5.3$ (Fig. 6b) and uplift pipe movement in dry sand for $\mathrm{H}_{\mathrm{c}} / \mathrm{D}$ $=4$ (Fig. 6c). A detailed comparison of test and analytical results with additional data and description of the modeling are provided by Jung et al. (2013a, 2013b).

Figure 7 presents plots of the maximum dimensionless lateral pipe force, $\mathrm{N}_{\mathrm{qH}}$, vs dimensionless depth, $H_{c} / D$, for dry medium, dense, and very dense sand for $H_{c} / D \leq 11$, based on both the largescale test results and analytical results provided by Jung, et al. (2013b). Soil properties for medium, dense, and very dense sand are summarized in Table 2. The dimensionless maximum pipe force is expressed as $\mathrm{F} /\left(\mathrm{H}_{\mathrm{c}} \bar{\gamma} \mathrm{DL}\right)$ for which $\mathrm{F}=$ soil-pipe reaction force, $\bar{\gamma}=$ soil unit weight contributing to effective stress at the pipe centerline, $\mathrm{H}_{\mathrm{c}}=$ depth to pipe center, $\mathrm{D}=$ outside pipe diameter, $\mathrm{L}=$ pipe length. Figure 7 also contains a plot of $\mathrm{N}_{\mathrm{qH}} \mathrm{vs} \mathrm{H}_{\mathrm{c}} / \mathrm{D}$ for partially saturated medium dense sand as reported by Jung et al. (2013b).

Pipelines are most often constructed in unsaturated soils, where a meniscus forms between soil particles, creating suction that binds the particles together. A direct comparison of $\mathrm{N}_{\mathrm{qH}}$ for dry and partially saturated medium sand in Figure 7 provides an estimate of the increased lateral pipe force related to suction. The plots for dry medium sand and partially saturated medium sand were developed from multiple large-scale soil-pipe interaction test results and supporting numerical simulations for sands with very similar dry unit weight (same density), minerology, angularity, and grain size distribution. Hence, the difference between the dimensionless lateral pipe force for partially saturated sand and dry sand represents the increase in horizontal force due to soil suction. Based on the data presented in Figure 7, suction contributed to an increase of about 15$20 \%$ in lateral load relative to dry sand conditions.

It should be noted that the increased percentage load applies for soil constituents, grain characteristics, and saturation conditions (water content $=4 \%$ and degree of saturation $=18 \%$ ) pertaining to the sand used in the large-scale soil-pipe loading experiments. Different soil characteristics, however, will result in different suction effects. Partial saturation and suction effects will increase soil reaction loads on buried pipelines relative to the effective stress 
conditions assumed in current design, which are associated with dry or fully saturated soil below the groundwater table. Thus, the influence of unsaturated soils on the reaction forces imposed on pipelines by ground deformation is an important topic for future research.

Figure 8 presents plots of the peak dimensionless uplift pipe force, $\mathrm{N}_{\mathrm{qUV}}$, vs. dimensionless depth, $H_{c} / D$, for dry medium and very dense sand for $H_{c} / D \leq 13$, based on the large-scale test and analytical results provided by Jung, et al. (2013a). The analytical model developed by Jung et al. (2013a) accounts for soil migration beneath the pipe through FE mesh adjustment coordinated with upward pipe displacement. For $\mathrm{H}_{\mathrm{c}} / \mathrm{D} \leq 5.5$, the model predicts decreasing uplift force after a peak value as upward movement of the pipe continues, which is consistent with experimental measurements. Similar to the comparison of analytical and experimental results for lateral soilpipe interaction, a systematic comparison of FE model results with multiple full-scale test measurements of pipe response to uplift displacement in dry sand show excellent agreement both with respect to maximum force and force-displacement relationships as presented by Jung, et al. (2013a).

\section{Vertical Downward Soil-Pipe Interaction}

Because large-scale 2D experimental results are not available for downward pipe movement in soil, FE analyses were used to simulate this mode of soil-pipe interaction. The FE modeling approach, which is described in detail by Jung (2010), was validated by simulating the vertical load response of deep foundation bearing elements and confirming that they compare favorably with the tip resistances of similar deep foundations as calculated from widely used bearing capacity formulations (e.g., Vesić, 1975; Kulhawy et al., 1983).

Approximately 1500 to 5000 elements were used in each $\mathrm{FE}$ simulation, depending on $\mathrm{H}_{\mathrm{c}} / \mathrm{D}$ conditions. Infinite elements (Zienkiewicz et al., 1983), were used to represent a semi-infinite soil medium, and the pipe was modeled as a rigid cylinder, with a soil/pipe interface friction

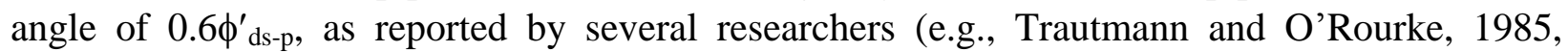
Trautmann et al., 1985). Pipe movement was generated by imposing vertical downward displacements on all pipe nodes. Separation and slip between soil and pipe were allowed in the analysis, and geostatic loading under $K_{0}=1$ conditions was applied to the soil and pipe at the beginning of each FE simulation. Methods recommended for the interpretation of deep and shallow foundation load tests (Akbas and Kulhawy, 2009a) were used to identify the maximum vertical force from the load vs settlement results for each simulation.

Figure 9 presents the $\mathrm{FE}$ results for the dimensionless maximum vertical downward force $\left(\mathrm{N}_{\mathrm{qVD}}\right)$ related to medium, dense, and very dense sand for $H_{c} / D=2-11$. As $H_{c} / D$ decreases, $N_{q V D}$ approaches a value analogous to the bearing capacity of a circular dished foundation with width $\mathrm{D}$ at the ground surface.

Guidelines for the seismic design of oil and gas pipelines recommend that the maximum vertical force associated with downward pipe movement be estimated by conventional bearing capacity formulations (ASCE, 1984; Honegger and Nyman, 2004). It is of interest therefore to compare the maximum vertical pipe force from conventional bearing capacity theory with the peak pipe force given by the FE simulations. 
The pipe bearing capacity was calculated according to widely used bearing capacity formulations (Vesić, 1975; Kulhawy et al., 1983; Akbas and Kulhawy, 2009b), which show that the maximum tip resistance of a deep foundation, $\mathrm{Q}_{\mathrm{tc}-\mathrm{bc}}$, can be estimated as

$$
\mathrm{Q}_{\mathrm{tc}-\mathrm{bc}}=\left(q N_{q} \xi_{q r} \xi_{q s} \xi_{q d} \xi_{q i} \xi_{q g} \xi_{q t}\right) A_{t i p}
$$

in which $q=$ vertical effective stress at $\mathrm{H}_{\mathrm{c}}, N_{q}=$ bearing capacity factor, $\xi_{q r}=$ rigidity factor, $\xi_{q s}$ $=$ shape factor, $\xi_{q d}=$ depth factor, $\xi_{q i}=$ load inclination factor, $\xi_{q g}=$ ground surface inclination factor, $\xi_{q t}=$ tilt of foundation factor, and $\mathrm{A}_{\text {tip }}=$ area of the base of the foundation. For this study, the $\xi_{q i}, \xi_{q g}, \xi_{q t}$ factors were equal to 1.0 because all analyses were conducted for a horizontal ground surface without load inclination or eccentricity.

The bearing capacity forces, $\mathrm{Q}_{\mathrm{tc}-\mathrm{bc}}$, were calculated with Eq. 10 for medium, dense, and very dense sand at $\mathrm{H}_{\mathrm{c}} / \mathrm{D}=8,11,15,20$, and 30 . The maximum bearing force from each $\mathrm{FE}$ analysis, $\mathrm{Q}_{\mathrm{tc}-\text { fea }}$, is plotted with respect to $\mathrm{Q}_{\mathrm{tc}-\mathrm{bc}}$ for $\mathrm{H}_{\mathrm{c}} / \mathrm{D}=8-30$ in Figure 10. As shown in the figure, the maximum bearing force from the 2D FE analyses is considerably lower than the bearing capacity calculated by conventional bearing capacity equations. As shown in Figure 8 , $\mathrm{Q}_{\text {tc-fea }}$ is approximately $1 / 3$ of $\mathrm{Q}_{\mathrm{tc}-\mathrm{bc}}$. This reduction in vertical bearing force for underground pipe relative to the conventional bearing capacity of foundations is corroborated by centrifuge test measurements reported by Xie et al. (2013), who report that the maximum vertical reaction force measured for underground pipe subjected to normal fault movement was between 1/3 and 1/10 the force derived from conventional bearing capacity formulations.

It should be noted that the downward movement of pipe, which is located below the ground surface, is accompanied by soil migration into a zone in the soil mass directly above the pipe. Such movement is markedly different from what occurs during deep foundation settlement, when soil migration cannot occur into a zone immediately above the base of the foundation. As a result, there are substantially higher constraints on soil deformation with higher resistance to penetration of the soil mass experienced by a conventional foundation.

\section{Simulation of Coupled Normal and Frictional Forces}

As explained previously, at-rest conditions are disrupted when a pipeline or conduit is intersected by soil movement. In response to large ground deformation, an underground pipeline is subjected to frictional force directly related to the soil reaction force normal to the pipeline longitudinal axis. Figure 4 shows that the apparent frictional force, $f_{A}=p_{H} \tan \delta$, will provide a close estimate of the actual frictional force, $f_{T}$, when $\tan \delta$ is between 0.4 and 0.6 , and will provide a conservative estimate when $\tan \delta>0.6$. To account for elevated frictional resistance as $p_{H}$ increases, the FE simulation must couple the normal and frictional forces. The need for coupling normal and frictional forces applies for lateral, upward, and downward pipe displacement.

Figure 11a shows a schematic of the 2D FE model, which was developed in this work with the software ABAQUS (2014) to account for coupled normal and frictional forces. The pipeline is represented by beam elements (type b33) and the soil resistance normal to the pipeline axis by 
nonlinear springs (type spring2). The springs are connected to the pipeline with uniaxial gap elements (type gapuni), which are interface elements allowing for the transfer of forces parallel and perpendicular to their axes. The normal force per unit distance, $p_{N}$, transferred through the gap element is controlled by a simple Coulomb friction law, $p_{N} \tan \delta$, so it is proportional to the normal force acting on the pipeline at each level of deformation.

The gap elements transfer forces only when the corresponding normal springs carry compressive forces. This is achieved by allowing separation of the gap elements when tensile normal forces are activated in response to load relaxation and separation between soil and pipe. Such modeling is important for simulating pipeline response to normal faulting where considerably different soil reaction forces are mobilized on the upthrown and downthrown sides of the fault. It is also important for simulating the effects of differential settlement and horizontal soil movement.

As shown in Figure 11b, pipeline joints can be modeled by rotational, transverse, and axial springs with linear, multi-linear, or non-linear characteristics developed from large-scale laboratory test results. Typically, the transverse spring stiffness would be set to a very high value, which simulates the contact within joints that governs their transverse shear behavior in the field. Soil reaction forces perpendicular and parallel to the pipe longitudinal axis are modeled by springs or spring-like relationships that account for linear, multi-linear, or non-linear interaction with the pipe.

There are several advantages to this modeling approach. It accounts simultaneously for axial and flexural pipe response as well as rotation and axial movement at pipeline joints. In this work the force-displacement relationships both normal and parallel to the pipeline longitudinal axis are developed from large-scale and centrifuge soil-pipeline test results (e.g., O'Rourke et al., 2008; Jung et al., 2013a and b) and validated against the results of large-scale and centrifuge tests of pipeline response to ground rupture performance (e.g., Ha et al., 2008; O'Rourke, 2010).

In the FE model, as an initial step, the displacements required to activate the normal forces for at-rest conditions are imposed on the transverse springs. With this adjustment, a longitudinal frictional force, consistent with that given by Eqn. 1, is activated when there is relative soil displacement along the pipeline, even in the absence of normal forces triggered by relative soil displacement normal to the pipe. During the simulation of ground deformation effects, incremental parallel and normal soil movements are applied simultaneously at the longitudinal and transverse spring nodes on both sides of the pipeline elements.

\section{Simulation of Pipeline Response to Strike-Slip Fault Movement}

Large-scale tests are described by O'Rourke et al. (2008) in which high density polyethylene (HDPE) pipelines were subjected to $1.22 \mathrm{~m}$ of strike-slip displacement at a vertical fault crossing angle of $65^{\circ}$, which is illustrated in Figure 12a. As previously described, the tests were performed on nominal $250-\mathrm{mm}$ and $400-\mathrm{mm}$ diameter pipelines at about $1-\mathrm{m}$ depth to pipe centerline. The outside diameter was $272 \mathrm{~mm}$ and $407 \mathrm{~mm}$ for the $250-\mathrm{mm}$ and $400-\mathrm{mm}$ pipe, respectively. The wall thickness was $24.9 \mathrm{~mm}$ for both. The 13.2-m-long, 3.2-m-wide and 2.3-mhigh split-basin test facility can generate maximum fault displacements of 1.2 to $1.8 \mathrm{~m}$. All splitbasin tests were conducted with partially saturated sand with dry unit weight and moisture 
content of $15.7 \mathrm{kN} / \mathrm{m}^{3}$ and $4 \%$, respectively.

Pipeline response to strike-slip faulting was simulated with an FE model composed of 43 beam elements (type b33), 168 spring elements (type spring2), and 84 gap elements (type gapuni). Strain rate and temperature effects of the HDPE were taken into account with stress vs. strain data from direct tension tests in an environmental chamber under constant temperature and strain rates. The stress vs strain results for a strain rate of $10 \% / \mathrm{min}$. at $21^{\circ} \mathrm{C}$ was found to match best the actual strain rate $(2 \% / \mathrm{min})$ and temperature $\left(26^{\circ} \mathrm{C}\right)$ associated with the full-scale tests. The analyses were performed with maximum soil forces for partially saturated sand in Figure 7 and bilinear force vs. displacement relationships as described by Jung et al. (2013a, 2013b).

As illustrated in Figure 12b, the orientation of the pipeline near the fault changes with respect to the direction of soil movement as the strike-slip offset increases. Figure $12 \mathrm{~b}$ shows how the initial relative normal and parallel soil displacements, $\delta_{N}$ and $\delta_{P}$, respectively, change as the orientation $\alpha$, of a pipeline segment alters from its initial orientation at $\alpha=0$. The figure shows how the initial relative displacements, $\delta_{N}$ and $\delta_{P}$, are decomposed into directions normal and parallel to the pipe to provide the relative normal and parallel displacement $\delta_{N \alpha}$ and $\delta_{P a}$, respectively, at any pipe segment orientation, $\alpha$. If $\tan \psi_{\alpha}=\delta_{N \alpha} / \delta_{P \alpha}$, then

$\tan \psi_{\alpha}=\frac{\delta_{N} \cos \alpha-\delta_{P} \sin \alpha}{\delta_{N} \sin \alpha+\delta_{P} \cos \alpha}$

which is simplified as

$$
\tan \psi_{\alpha}=\frac{\tan \psi-\tan \alpha}{\tan \psi \tan \alpha+1}
$$

from which

$$
\psi_{\alpha}=\tan ^{-1}\left(\frac{\tan \psi-\tan \alpha}{\tan \psi \tan \alpha+1}\right)
$$

where $\tan \psi$ is the ratio of $\delta_{N} / \delta_{P}$ at each pipeline node in the numerical simulation and $\psi_{\alpha}$ is the angle between the frictional force vector and the longitudinal axis of the pipe as its orientation, $\alpha$, changes. The angle, $\psi_{\alpha}$, is the angle of obliquity between the frictional force vector and the pipe longitudinal axis. Using the 2D FE modeling described in the previous section, one can obtain both $\tan \psi$ and $\tan \alpha$ at each pipeline node at each increment of fault movement during the numerical simulation and calculate $\psi_{\alpha}$ with Eq. 13. At the location of the intersection of the pipeline with the fault plane, please note that $\tan \psi=\tan \beta$, where $\beta$ is the pipeline/fault angle of intersection.

Treating friction as a unit vector, $\cos \psi_{\alpha}$ is the fraction of the total frictional force, $f_{T}$, along the pipeline longitudinal axis. Because the FE model does not account for the change in frictional force along the longitudinal axis of the pipe as the pipeline orientation changes with increasing fault offset, Eqn. 13 was used to estimate this effect and assess its influence on the axial strain 
mobilized in the pipeline.

Pipeline response to fault displacement was simulated by assuming initially that the full frictional force was directed along the longitudinal axis of the pipeline, corresponding to an angle of obliquity equal to zero. Values of $\psi_{\alpha}$ and $\cos \psi_{\alpha}$ were calculated at each pipeline node at each increment of strike-slip movement through the use of Eq. 13. The average of $\cos \psi_{\alpha}$, over all the displacement increments until the maximum strike slip displacement, was then assigned at each FE pipe node to estimate the effect of reduced longitudinal frictional force. Outside the zone of changing $\alpha$, at-rest conditions were assumed in accordance with Eq. 1 .

Figure 13 compares the measured axial strains with FE results for three cases, including the atrest conditions with no coupling between normal and frictional forces (denoted as " $\mathrm{K}_{0}$ Conditions" in the figure). The second case pertains to simulation with coupled normal and frictional forces (denoted as "Coupled"), where the apparent frictional force, $\mathrm{f}_{\mathrm{A}}=p_{H} \tan \delta$, was used with Eq. 9 to estimate frictional force in the absence of orientation and obliquity corrections. Tan $\delta=0.44$ was used in accordance with HDPE/sand direct shear measurements reported by Olson (2009). This case assumes that the full frictional force is directed along the longitudinal pipeline axis. The third case pertains to coupled normal and frictional forces with corrections for frictional force obliquity (denoted as "Coupled Orientation \& Obliquity Correction").

As shown in Figure 13 the measured axial strains agree most closely with the coupled FE simulations and show that the maximum axial strain is underestimated by over $10 \%$ with the FE simulation that accounts only for $\mathrm{K}_{0}$ conditions. The closest agreement between the experimental and analytical results applies for the coupled FE simulations with and without orientation and obliquity corrections, The maximum simulated strain is approximately $6 \%$ and $10 \%$ higher than the maximum measured value for coupled analysis with and without obliquity corrections, respectively. The frictional force obliquity correction has a relatively small effect on the analytical outcome compared to the case without such correction. It decreases the maximum axial strain by less than $4 \%$ relative to the maximum axial strain obtained with the frictional force directed along the pipeline longitudinal axis.

The differing approaches to modeling longitudinal friction have little effect on bending strains, which are highly dependent on $\mathrm{p}_{\mathrm{H}}$ modeling that was the same for all FE results. In general, the differences among the analytical and measured bending strains are less than 5\%. Analytical and measured bending strains are plotted and discussed in conjunction with Figure 14 in the next section of this paper.

\section{Analytical and Experimental Results for Strike-Slip Fault and Normal Fault Movements}

Simulations of soil-pipeline interaction under strike-slip and normal fault displacements were performed using coupled normal and frictional forces. Corrections for pipe orientation near strike-slip faulting were not used because these adjustments have a relatively minor effect on the analytical results, as discussed above. The numerical results are presented for lateral force vs. displacement relationships presented by Jung (2010) and Jung et al. (2013b) with maximum lateral loads for both dry and partially saturated medium dense sand taken from Figure 7. The 
numerical results are compared with pipeline strain measurements reported by O'Rourke at al. (2008) from large-scale strike-slip soil movements on HDPE pipelines conducted in the splitbasin test facility at Cornell University. The numerical results for normal faulting are compared with the results of a centrifuge test at RPI described by Ha (2007) and Xie at al. (2013). The experimental conditions, including soil properties, pipeline characteristics, and split-basin dimensions, are described in the previous section of this paper. Likewise, the characteristics of the finite element model are described in the previous section.

Figures $14 \mathrm{a}$ and $\mathrm{b}$ compare the numerical and measured axial and bending strains for large-scale tests on a 250-mm and two 400-mm HDPE pipelines, respectively, at $1.22 \mathrm{~m}$ of strike-slip displacement. Axial strains are calculated as the average pipe crown and invert strains, and bending strains are determined as one half the difference between the springline strains. Insert diagrams in the figure show the crown, springline, and invert locations. There was a short distance along the pipeline $(\sim 1 \mathrm{~m})$ where a tactile pressure sensor was installed and strain gages were absent, as shown in Figure 14a.

The numerical and experimental bending strains follow similar distributions, with the maximum experimental bending strains best matched by analytical results that account for suction. The numerical and experimental axial strains likewise follow similar distributions, with the maximum FE axial strain for dry and partially saturated conditions between 5 and $10 \%$ higher, respectively, than the measured axial strain.

Figure 14b presents the measured and simulated strains for two 400-mm-diameter HDPE pipelines. Several tactile pressure sensors were installed south of the fault rupture, as shown in the figure. A double layer of Teflon sheets was used to protect the sensors, and resulted in a sizeable zone where very low shear stress was transmitted to the sensor and pipe. Reduced longitudinal friction in this zone was modeled by $\tan \delta=0.1$ - to represent the effect of the Teflon sheets. With nearly half the pipeline south of the ground rupture isolated from the full effects of soil shear forces, additional load was conveyed asymmetrically to each end of the test pipelines, with higher axial strains at the south end. This asymmetric distribution of axial strain is well represented by the FE results. Measured strains at the center of the pipeline appear to be influenced by the adjacent tactile pressure sensor, but otherwise are predominantly within $10 \%$ of the analytical values.

Figures $14 \mathrm{c}$, d, and e compare FE and centrifuge measured strains for a prototype 400-mmnominal-diameter HDPE pipeline subject to normal fault displacement. Numerical simulation of the centrifuge test with 2D and 3D FE soil-pipeline interaction models is described in detail by Xie et al. (2013). Centrifuge testing was performed at $12.2 \mathrm{~g}$ with a split-box container fabricated for in-flight simulation of fault rupture (Ha, 2007). A maximum prototype displacement of 0.48 $\mathrm{m}$ was imposed as abrupt vertical movement of one side of the container relative to the other (see insert diagram in Figure 14e). The prototype depth to pipe centerline was approximately $1.2 \mathrm{~m}$ in partially saturated sand with dry unit weight and moisture content of $14.7 \mathrm{kN} / \mathrm{m}^{3}$ and $4 \%$, respectively. The centrifuge tests were conducted with the same HDPE properties, diameter, and thickness as the pipe in the large-scale Cornell experiments described above.

The 2D FE model used in this work to simulate the experiment was composed of 47 beam 
elements (type b33), 184 spring elements (type spring2) and 96 gap elements (type gapuni) that account for coupled normal and frictional forces. The stress vs. strain relationship for the HDPE behavior corresponding to $1 \%$ loading rate at $21^{\circ} \mathrm{C}$, as reported by $\mathrm{Ha}$ (2007) was used in the simulations. The analysis was performed with uplift and vertical downward forces from Figures 6 and 9 for medium sand and bilinear force vs. displacement relationships (Jung, 2010; Jung at al., 2013a). Because the burial depth was relatively shallow with $\mathrm{H}_{\mathrm{c}} / \mathrm{D}=2.7$, uplift forces decreasing after a peak force were modeled in accordance Jung et al. (2013a). The vertical displacement was distributed linearly from the edge of the Rankine active zone on the upthrown side of the fault (see insert diagram in Figure 14c) to the maximum downward movement at the fault plane. For the reported $40^{\circ}$ angle of soil shear resistance (Ha, 2007), the Rankine zone extends about $0.6 \mathrm{~m}$ from the fault plane.

Figures 14c, d, and e compare FE results with measured crown, invert, and bending strains, respectively, at $0.48 \mathrm{~m}$ of normal fault displacement for 1) 2D FE model (denoted as "2D Beam" in the figure), 2) 3D FE model with pipe shell elements and nonlinear springs for soil-pipe interaction (denoted as "3D Shell"), and 3) 3D FE model with pipe shell and continuum soil elements (denoted as "3D Cont."). The FE results for the 3D model are presented in the same format used by Xie et al. (2013).

All FE results show distributions similar to the measured ones. Both the 3D Shell and 3D Cont. models underpredict the maximum crown strain by $40 \%$ and $28 \%$, respectively. In comparison, the 2D FE model underpredicts the maximum crown strain by $7 \%$. Overall the 2D FE model results are comparable to the $3 \mathrm{D}$ model results, with maximum strains that are more consistent with measured maximum strains than those of the 3D models.

This finding differs from the conclusions presented by Xie et al. (2013) who found that 2D FE model predictions did not agree well with the measured strains. The 2D FE models applied in this work incorporate improvements with respect to the ASCE (1984) recommended soil-pipe interaction modeling procedures that were followed by Xie et al. (2013). The 2D FE model results in Figures 14c, d, and e incorporate the findings presented in this paper, which differ from the 2D FE modeling procedures followed by Xie at al. (2013) in four ways. First, coupled normal and frictional forces were modeled in the 2D FE analysis, using the FE setup in Figure 9 in contrast to the at-rest $\left(\mathrm{K}_{0}\right)$ conditions for longitudinal friction applied by Xie et al. (2013). Second, the 2D FE modeling used vertical downward forces from Figure 9 which are about 1/3 the magnitude derived from the conventional bear capacity approach presented in ASCE (1984). Reduction in the vertical downward soil reaction force is consistent with the findings of Xie et al. (2013) who recommend decreasing the vertical soil spring strength by a factor of $1 / 3$ to $1 / 10$ for 2D FE modeling of normal fault interaction with pipelines. Third, uplift forces decreasing after a peak force were modeled following the recommendations of Jung et al. (2013a). Fourth, a linear distribution of vertical displacement from the fault rupture to the end of the Rankine active zone on the upthrown side of the fault was used instead of abrupt vertical movement. The Rankine zone, over which the fault offset was linearly distributed, is shown in the inset illustration of Figure 14c. This displacement pattern agrees with observations of surficial ground movement after the centrifuge test (Ha, 2007) and is consistent with active soil conditions that are mobilized near the upthrown side of a normal fault. 


\section{Conclusions}

The principal causes of earthquake-induced ground deformation are identified and their interaction with pipelines and conduits is described. Methods for modeling soil-pipe interaction are reviewed with concentration on lateral, uplift, and vertical downward relative movement between pipe and soil. The fundamental mechanics of soil-pipe interaction are investigated through normal surface stresses measured with tactile pressure sensors during large-scale tests and the resolution of those stresses for normal and lateral force characterization. A procedure is developed for modeling coupled normal and longitudinal frictional forces on pipelines subject to permanent ground deformation, and a finite element configuration for coupled normal and frictional force modeling is presented. The effects of changing orientation between pipe and soil displacement at fault crossings and the margins of lateral spreads/landslides is investigated and quantified. Specific findings of interest are:

- Full-scale soil-pipe interaction tests show that suction associated with partially saturated sand contributed to an increase of about $15-20 \%$ in lateral pipe load relative to the load for dry sand conditions. Partial saturation and suction effects can increase lateral pipeline forces relative to those in dry soil or fully saturated soil below the groundwater table, depending on soil minerology and grain size characteristics.

- The maximum downward pipe force is only about one-third the maximum force determined with conventional bearing capacity equations. The conventional foundation bearing capacity forces currently recommended in design appear to be overly conservative.

- Coupling normal and frictional forces in the numerical modeling of soil-pipe interaction is important for assessing the maximum axial pipe strain. Neglecting this coupled interaction through the sole use of at-rest $\left(\mathrm{K}_{0}\right)$ conditions for evaluating frictional force leads to under-conservative results for axial strain.

- A correction factor is derived for converting the soil reaction force normal to the pipeline longitudinal axis to the soil reaction force normal to the circular pipe surface. Analytical modeling that accounts for this correction is shown to provide an estimate that is either accurate or slightly conservative for analysis and design.

The analytical results for pipeline response to strike-slip fault rupture using the models developed in this work compare favorably with the results of large-scale tests of strike-slip fault movement imposed on 250-mm and 400-mm-diameter HDPE pipelines as well as centrifuge testing of normal fault movement on a 400-mm HDPE pipeline.

\section{Acknowledgements}

The work on which this paper was based was supported by the National Science Foundation (NSF) under Grant No. CMMI-1041498. Any opinions, findings and conclusions or recommendations expressed in this material are those of the authors and do not necessarily reflect the views of the NSF.

\section{References}


ABAQUS. Theory Manual of ABAQUS. ABAQUS Inc.: Providence, RI, 2014.

Akbas SO, Kulhawy FH. Axial compression of footings in cohesionless soils. I: Load-settlement behavior. Journal of geotechnical and geoenvironmental engineering 2009a; 135(11): 1562-1574.

Akbas SO, Kulhawy FH. Axial compression of footings in cohesionless soils. II: Bearing Capacity. Journal of geotechnical and geoenvironmental engineering 2009b; 135(11): 1575-1582.

ASCE. Guidelines for the seismic design of oil and gas pipeline systems. Committee on Gas and Liquid Fuel Lifelines, American Society of Civil Engineers: Reston, VA, 1984.

Bird J, O'Rourke TD, Bracegirdle A, Bommer J, Tromans I. A. Framework for assessing earthquake hazards for major pipelines 2004, Proceedings, Intl. Conf. on Terrain and Geohazards Facing Onshore Oil and Gas Pipelines, London, $U K$.

Chapra SC, Canale RP. Numerical Methods for Engineers. McGraw Hill: New York, 2006.

Ha D, Abdoun T, O’Rourke MJ, Symans MD ,O'Rourke TD, Palmer MC, Stewart HE. Buried high-density polyethylene pipelines subjected to normal and strike-slip faulting - a centrifuge investigation. Canadian Geotechnical Journal 2008; 45(12):1733-1742.

Ha D. Evaluation of ground rupture effects on buried HDPE pipelines. PhD Thesis, RPI: Troy, NY, 2007.

Honegger D and Nyman DJ. Guidelines for the seismic design and assessment of natural gas and liquid hydrocarbon pipelines. Pipeline Research Council International; Catalog No. L51927, 2004.

Jung JK. Soil-pipe interaction under plane strain conditions. PhD Thesis, Cornell University: Ithaca, NY, 2010.

Jung JK, O'Rourke TD, Olson NA. Uplift soil-pipe interaction in granular soil. Canadian Geotechnical Journal 2013a; 50(7): 744-753.

Jung JK, O'Rourke TD, Olson NA. Lateral soil-pipe interaction in dry and partially saturated sand. Journal of Geotechnical and Geoenvironmental Engineering 2013b; 139(12): 2028-2036.

Kulhawy FH, Trautmann CH, Beech JF, O'Rourke TD, McGuire W, Wood WA, and Capano C. Transmission line structure Foundations for Uplift/Compression Loading. Electric Power Research Institute; Report EL-2870 1983: Palo Alto, CA.

Olson N. Soil Performance for large scale soil-pipeline tests. PhD Thesis, Cornell University: Ithaca, NY, 2009.

O'Rourke MJ and Liu J. The seismic design of buried and offshore pipelines. MCEER Monograph No. 4, 2012, MCEER, University at Buffalo, Buffalo, NY.

O'Rourke TD, Jezerski JM, Olson NA, Bonneau AL, Palmer MC, Stewart HE, O'Rourke MJ, Abdoun T. Geotechnics of pipeline system response to earthquakes. Geotechnical Earthquake Engineering and Soil Dynamics IV (GEESD) 2008: Sacramento, $C A$.

O’Rourke TD. Geohazards and large, geographically distributed systems. Geotechnique 2010; 60 (7): 505-543.

Palmer MC, O'Rourke TD, Olson NA, Abdoun T, Ha D, O'Rourke MJ. Tactile pressure sensors for soil - structure interaction assessment. Journal of Geotechnical and Geoenvir. Engineering 2009; 135(11): 1638 - 1645. 
Trautmann CH and O'Rourke TD. Lateral force-displacement response of buried pipe. Journal of Geotechnical Engineering 1985; 111(9): 1068-1084.

Trautmann CH, O'Rourke TD, Kulhawy FH. Uplift force-displacement response of buried pipe. Journal of Geotechnical Engineering 1985; 111(9): 1061-1076.

Vesić, A.S. Bearing capacity of shallow foundations, Chapter 3 in Foundation Engineering Handbook, 1975Ed. by H. Winterkorn and H.Y. Feng, Van Nostrand Reinhold Company, New York, NY, 1975, pp. 212-147.

Xie X, Symans MD, O'Rourke MJ, Abdoun TH, O'Rourke TD, Palmer MC, Stewart HE. Numerical Modeling of Buried HDPE Pipelines Subjected to Normal Faulting: A Case Study. Earthquake Spectra 2013; 29 (2): 609-632.

Zienkiewicz OC, Emson C, Bettess P. A novel boundary infinite element. International Journal for Numerical Methods in Engineering 1983; 19(3): 393-404.

\section{Appendix A: Regression of Normalized Pipe Surface Stress Measurements}

Curve fitting techniques described by Chapra and Canale (2006) for a sinusoidal function with angular frequency, $\omega_{0}$, were used to derive an analytical expression for the normal stresses distribution. The least squares model follows the expression

$$
y=A_{o}+A_{1} \cos \left(\omega_{o} \theta\right)+B_{1} \sin \left(\omega_{o} \theta\right)
$$

in which $A_{0}=\frac{1}{N} \sum_{i=1}^{N} y_{i}, A_{1}=\frac{2}{N} \sum_{i=1}^{N} y_{i} \cos \left(\omega_{o} \theta_{i}\right), B_{1}=\frac{2}{N} \sum_{i=1}^{N} y_{i} \sin \left(\omega_{o} \theta_{i}\right), \theta$ is the angular distance from the pipe crown, and $N$ is the number of equispaced data points. The alternative form for $\mathrm{Eq}$ (A.1) is

$$
y=A_{o}+C_{1} \sin \left(\omega_{o} \theta+\Delta\right)
$$

in which $\Delta=\varphi+\pi / 2, \varphi=\tan ^{-1}\left(-B_{1} / A_{1}\right)$, and $C_{1}=\left(A_{1}^{2}+B_{1}^{2}\right)^{1 / 2}$, which can be rearranged as

$$
\sin ^{-1}\left[\frac{y-A_{o}}{C_{1}}\right]=\omega_{o} \theta+\Delta
$$

When $A_{1}<0, \pi$ needs to be added to $\varphi$. 
Table 1: Summary of the principal causes and types of transient and permanent ground deformation associated with earthquakes (Bird et al., 2004)

\begin{tabular}{|c|c|c|}
\hline Type & Cause & Description \\
\hline \multirow{5}{*}{ Transient } & Travelling ground waves & $\begin{array}{l}\text { Near surface ground deformation caused by body waves propagating } \\
\text { from a seismic source. }\end{array}$ \\
\hline & $\begin{array}{l}\text { Surface Wave } \\
\text { Generation in Large } \\
\text { Sedimentary Basins }\end{array}$ & $\begin{array}{l}\text { Surface waves generated by scattering incoming waves in large } \\
\text { sedimentary basins typically several } \mathrm{km} \text { wide, with depths }<1 \mathrm{~km} \text {. }\end{array}$ \\
\hline & $\begin{array}{l}\text { Vibration of relatively } \\
\text { narrow soil-filled valleys }\end{array}$ & $\begin{array}{l}\text { Deformation of sediment-filled valleys with respect to relatively rigid } \\
\text { valley boundaries. Valley width and depths are typically several } \\
\text { hundred and several tens of meters, respectively. }\end{array}$ \\
\hline & Ridge shattering & $\begin{array}{l}\text { Ground disturbance along steep ridges and elevated topography that } \\
\text { may be accompanied locally by slip in fractured rock. }\end{array}$ \\
\hline & Ground oscillation & $\begin{array}{l}\text { Transient lateral shear strains and horizontal movement of liquefiable } \\
\text { soil relative to adjacent and underlying competent material. }\end{array}$ \\
\hline \multirow{5}{*}{ Permanent } & Faulting & $\begin{array}{l}\text { The principal components of fault movement include 1) strike, 2) } \\
\text { reverse, and 3) normal slip. Reverse and normal faults promote } \\
\text { compression and tension, depending on the angle of intersection } \\
\text { between lifelines and the fault trace. }\end{array}$ \\
\hline & $\begin{array}{l}\text { Tectonic Uplift and } \\
\text { Subsidence }\end{array}$ & $\begin{array}{l}\text { Regional changes in dimension associated with crustal deformation. } \\
\text { Deformation occurs over a long distance so strains imposed will be } \\
\text { small. Subsidence adjacent to water bodies can flood sections of a } \\
\text { lifeline and possibly lead to erosion and undermining. }\end{array}$ \\
\hline & Liquefaction & $\begin{array}{l}\text { Displacement caused by transformation of saturated, cohesionless soils } \\
\text { to liquefied state or condition of substantially reduced shear strength. } \\
\text { Liquefaction-induced lifeline deformation can be caused by 1) lateral } \\
\text { spread, 2) flow failure, 3) local subsidence, 4) post-liquefaction } \\
\text { consolidation, 5) buoyancy effects, and 6) loss of bearing. }\end{array}$ \\
\hline & Landslides & $\begin{array}{l}\text { Mass movement of the ground triggered by inertial forces from } \\
\text { seismic shaking. Many displacement patterns are possible. Principal } \\
\text { forms of movement include 1) rock falls, 2) relatively shallow } \\
\text { slumping and sliding of soil, and 3) relatively deep translation and } \\
\text { rotation of soil and rock. Landslides include lurching and soil block } \\
\text { movement in which ground displacements are triggered by transient } \\
\text { loading of gently sloping deposits underlain by weak soil not } \\
\text { susceptible to liquefaction. }\end{array}$ \\
\hline & Densification & $\begin{array}{l}\text { Decrease in volume caused by seismic vibration of dry or partially } \\
\text { saturated cohesionless soil. }\end{array}$ \\
\hline
\end{tabular}


Table 2. Summary of representative peak angles of shear resistance and angles of dilatency for medium, dense, and very dense sand

\begin{tabular}{|c|c|c|c|c|}
\hline Density & $\gamma_{\mathrm{d}}{ }^{1}\left(\mathrm{kN} / \mathrm{m}^{3}\right)$ & $\psi_{\mathrm{p}}{ }^{2}$ (degrees) & $\phi^{\prime}{ }_{\text {ds-p }}^{3}$ (degrees) & $\phi_{\text {ps-p }}^{\prime}{ }^{4}$ (degrees) \\
\hline Medium & $15.5-16.5$ & $3 \sim 6$ & $35 \sim 37$ & $41 \sim 44$ \\
\hline Dense & $16.5-17.5$ & $7 \sim 10$ & $38 \sim 40$ & $45 \sim 47$ \\
\hline Very Dense & $17.5-18.0$ & $11 \sim 17$ & $41 \sim 43$ & $48 \sim 49$ \\
\hline
\end{tabular}

$1-\gamma_{\mathrm{d}}=$ dry soil unit weight $\quad 3-\phi_{\text {ds-p }}^{\prime}=$ direct shear peak angle of shear strength

$2-\psi_{\mathrm{p}}=$ peak angle of dilatency $\quad 4-\phi_{\mathrm{ps}-\mathrm{p}}^{\prime}=$ plane strain peak angle of shear strength 


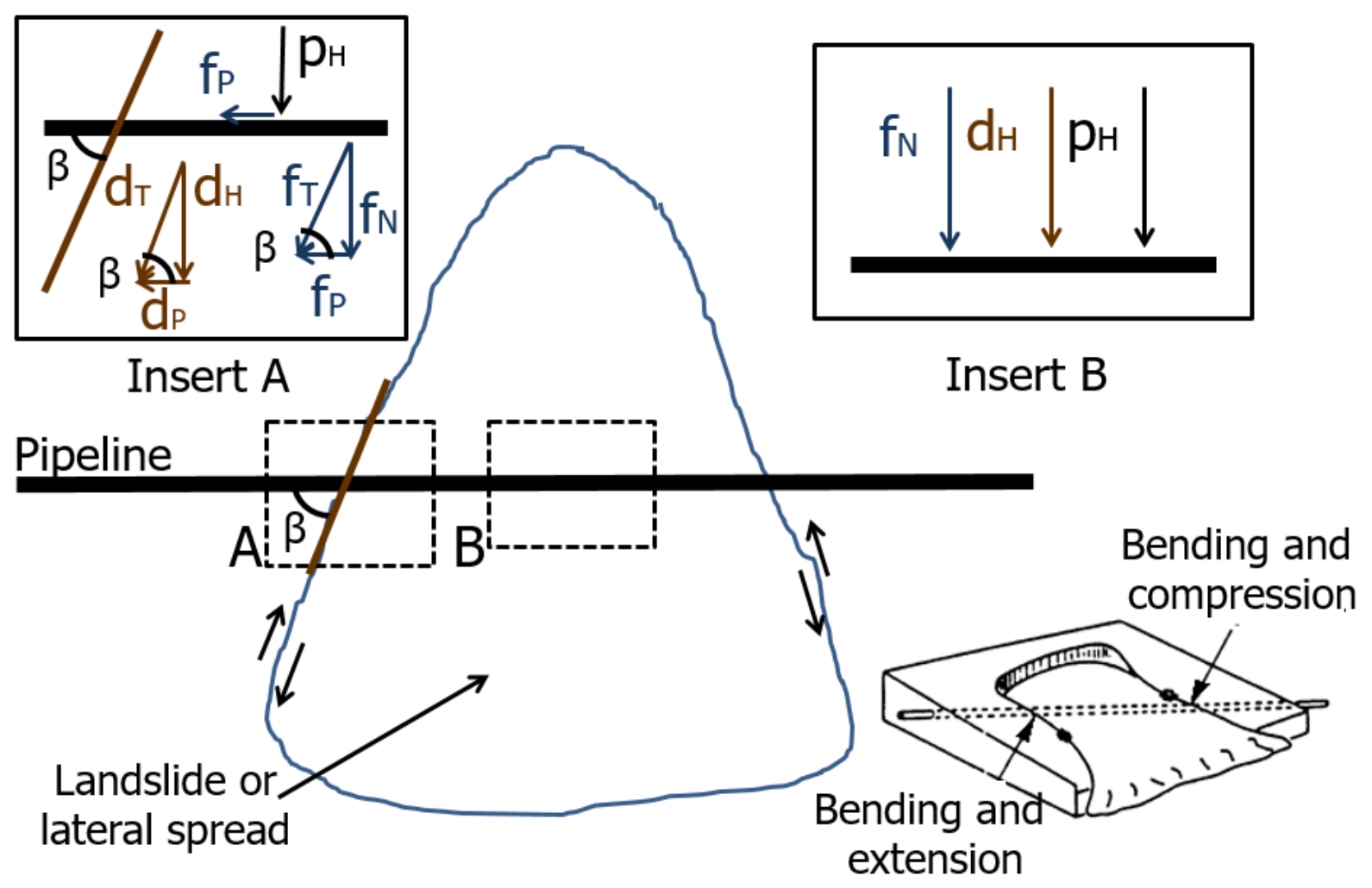

a) Plan View

b) 3D View

Figure 1. Plan and 3D views of pipeline intersection with landslide or lateral spread 


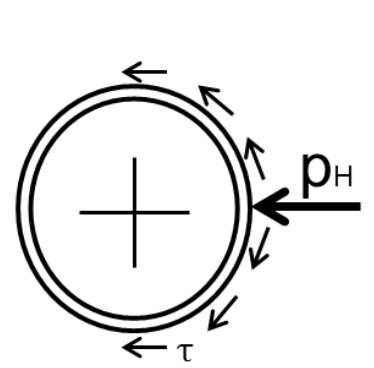

a) Shear Stress Orientation

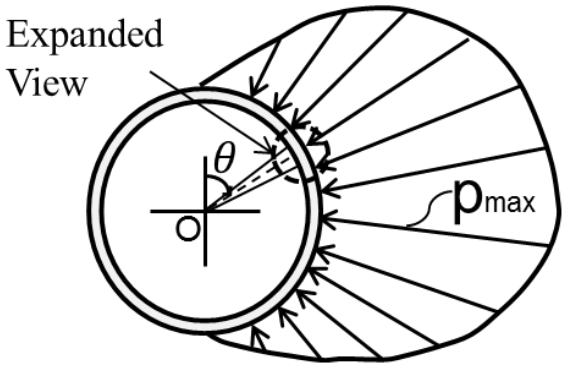

b) Normal Stress Distribution

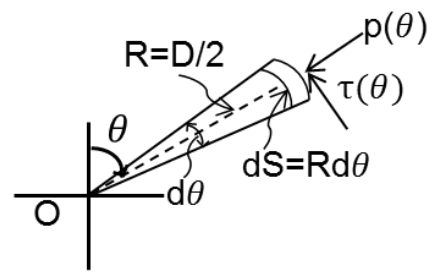

Note: Not in scale

c) Expanded View

Figure 2. Lateral soil- pipe interaction model for underground pipelines (Palmer et al., 2009) 

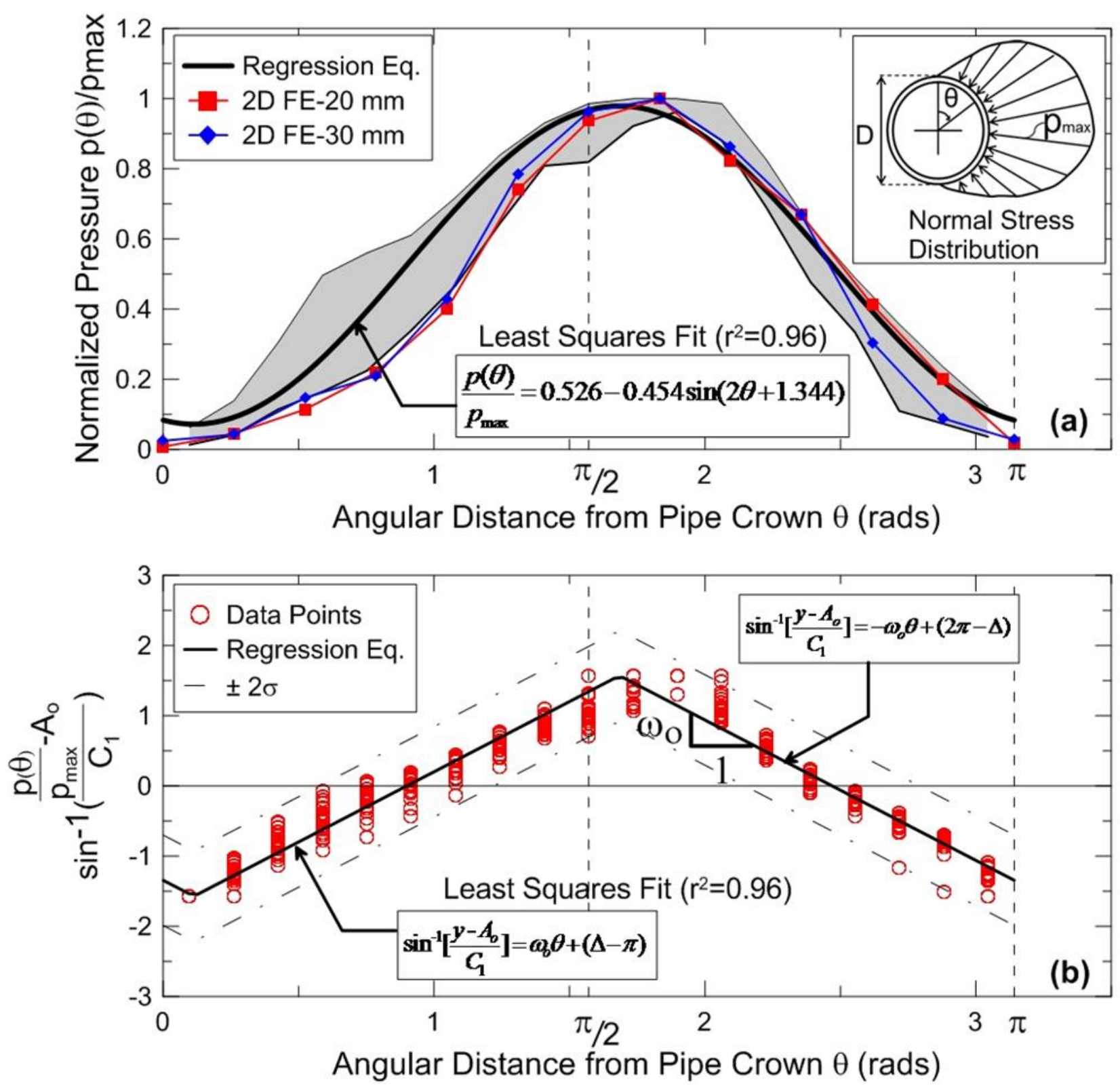

Figure 3. Normalized pressure vs. angular distance for (a) sinusoidal pressure distribution and (b) linear regression with transformed normalized pressure 

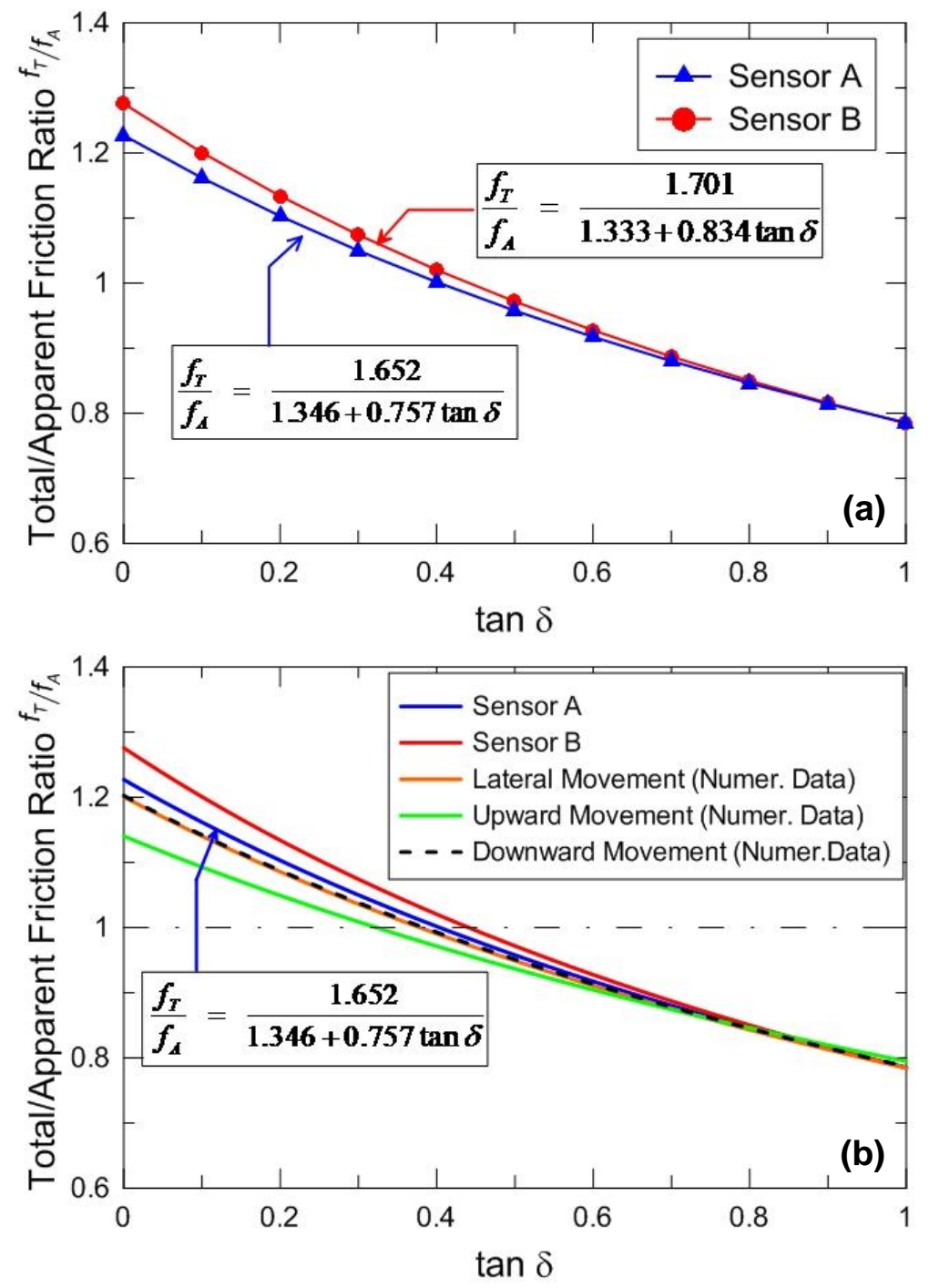

Figure 4. Ratio of total to apparent friction $f_{T} / f_{A} v s . \tan \delta$ based on (a) tactile pressure sensor measurements and (b) tactile pressure sensor and numerical data 


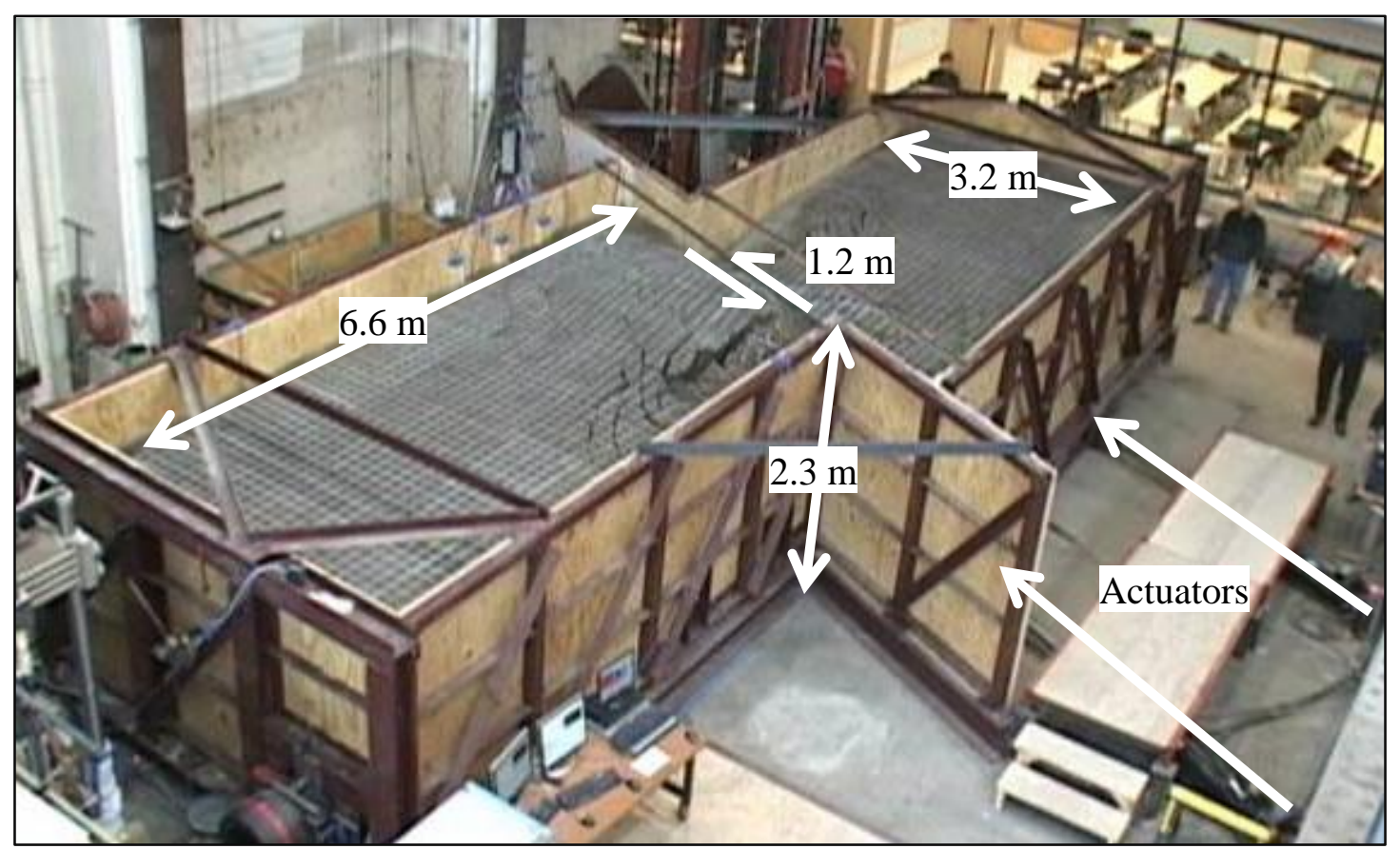

Figure 5. Large-Scale Split-Basin Test Facility at Cornell University 

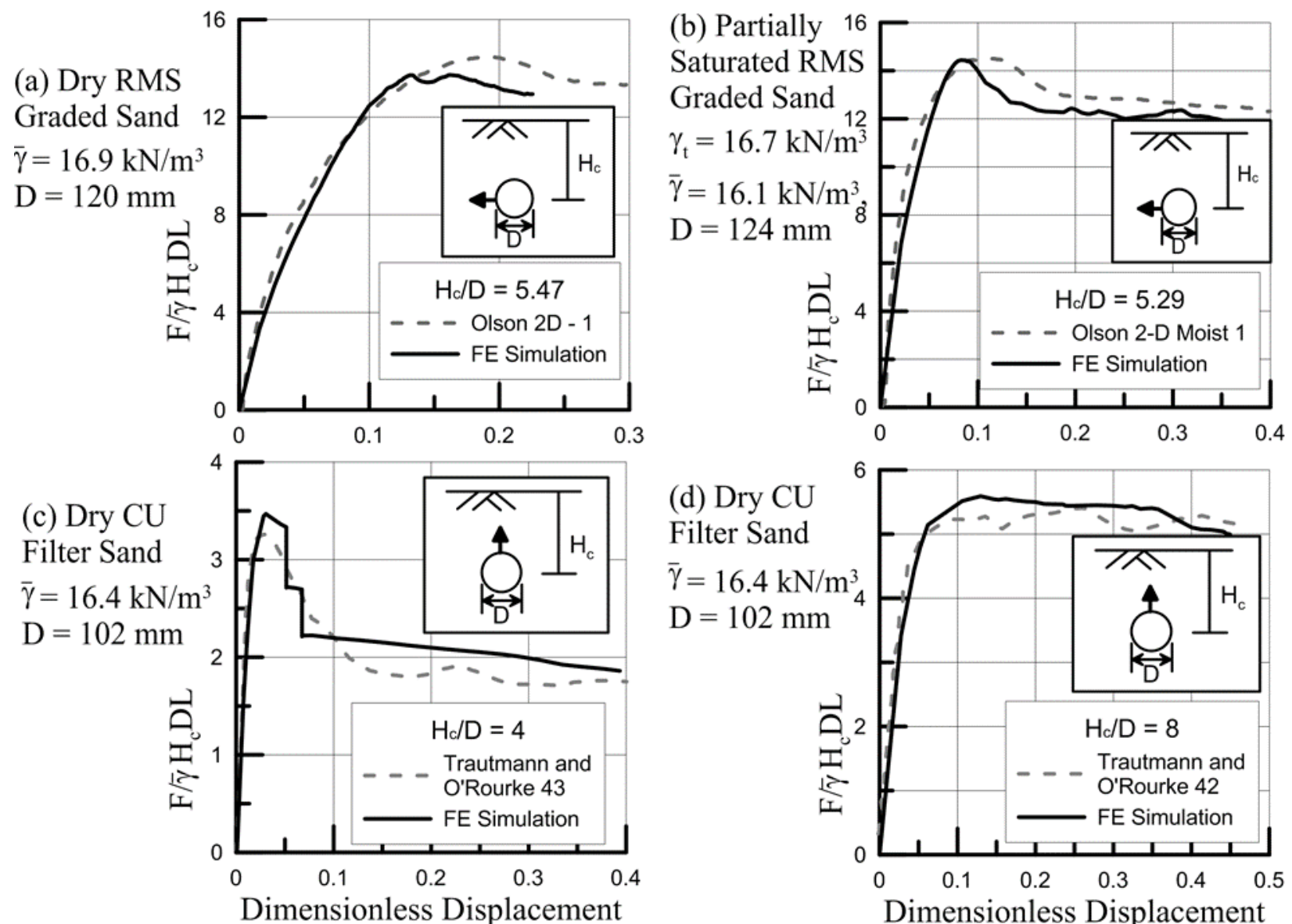

Figure 6. Comparison of Large-scale Test and Analytical Results for Lateral and Upward Movement of Pipe in Dry and Partially Saturated Sand (after Jung et al. 2013a and 2013b) 


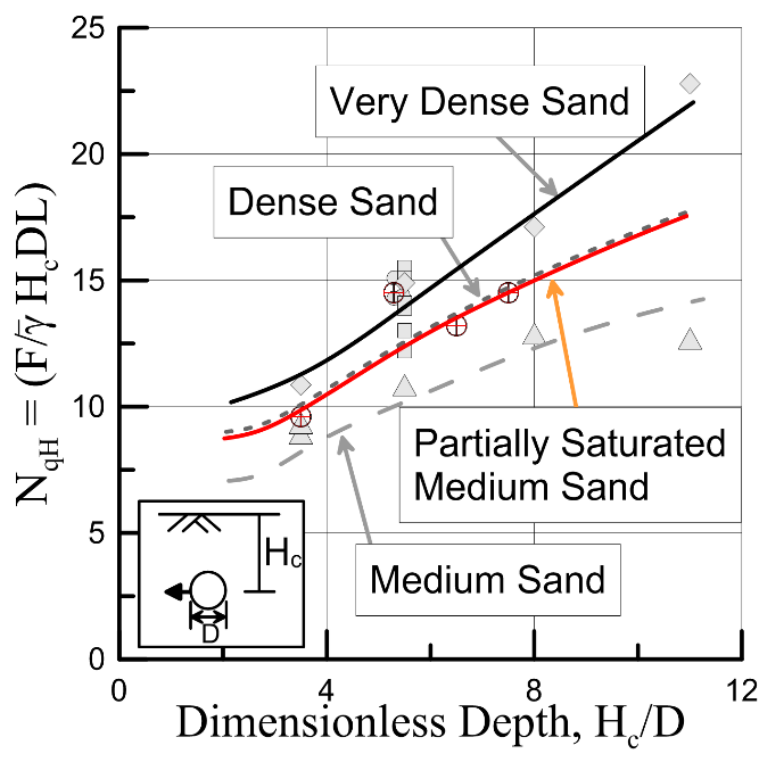

Note: For data sources, see Jung et al. (2013b)

Figure 7. Maximum dimensionless lateral pipe force 


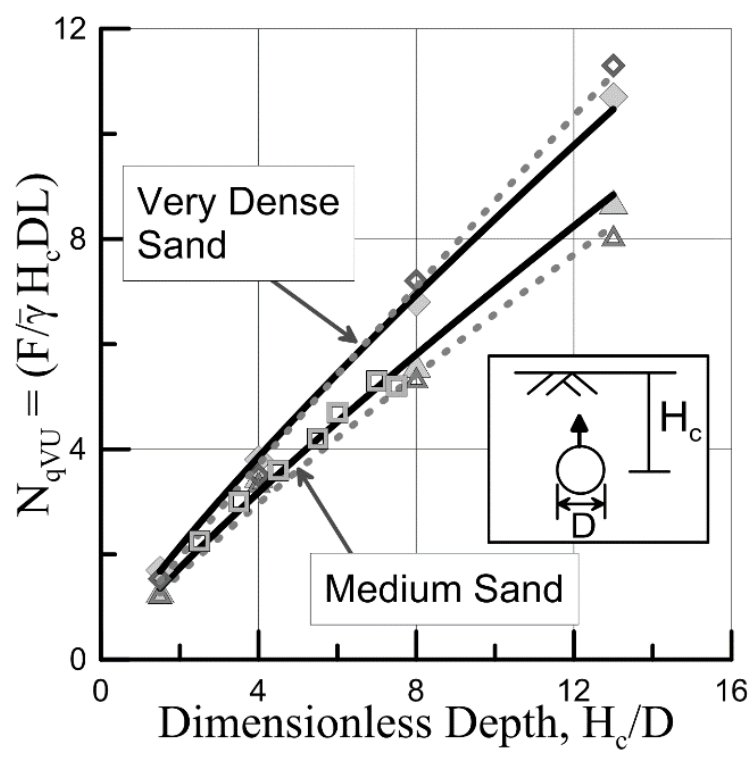

Note: For data sources, see Jung et al. (2013a)

Figure 8. Maximum dimensionless uplift pipe force 


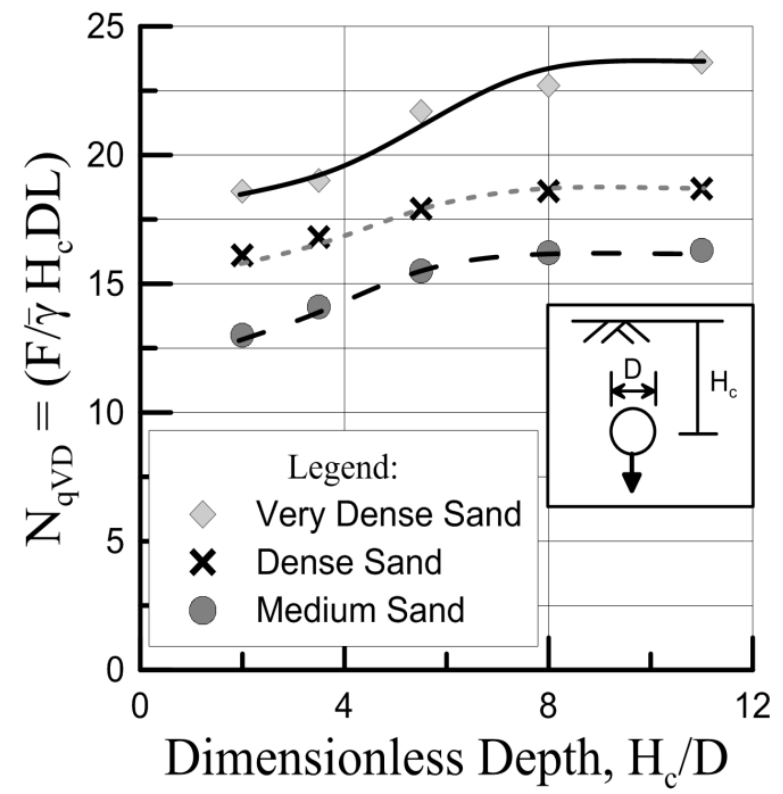

Figure 9. Finite element dimensionless maximum vertical downward force 


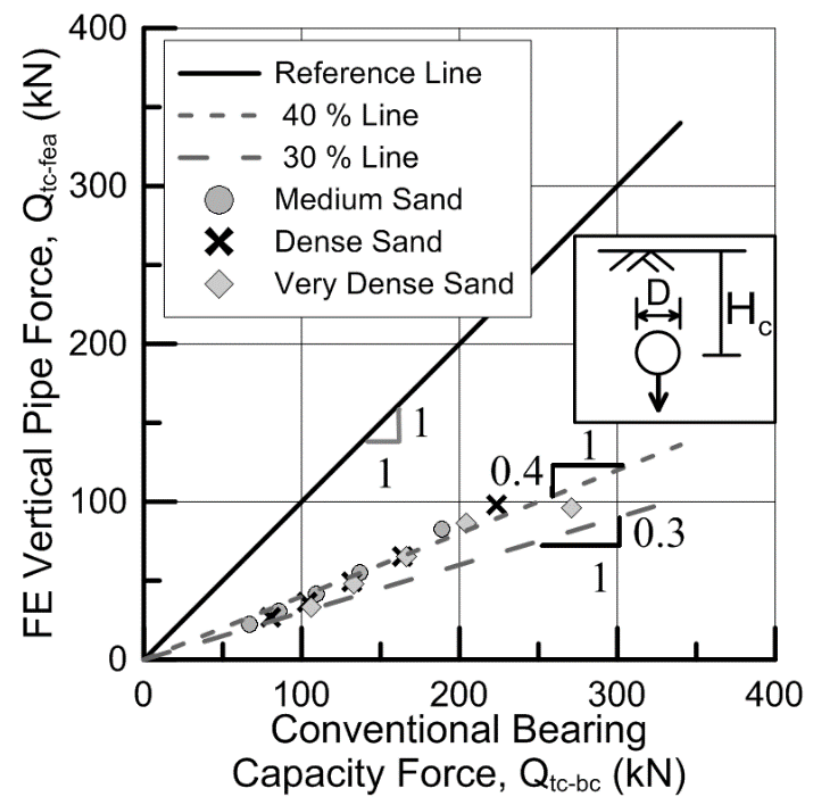

Figure 10. Finite element vertical force vs. bearing capacity vertical force 


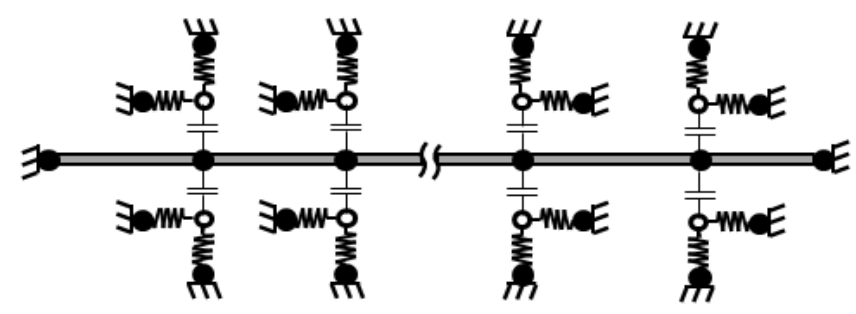

(a) Coupled model of soil-pipeline interaction
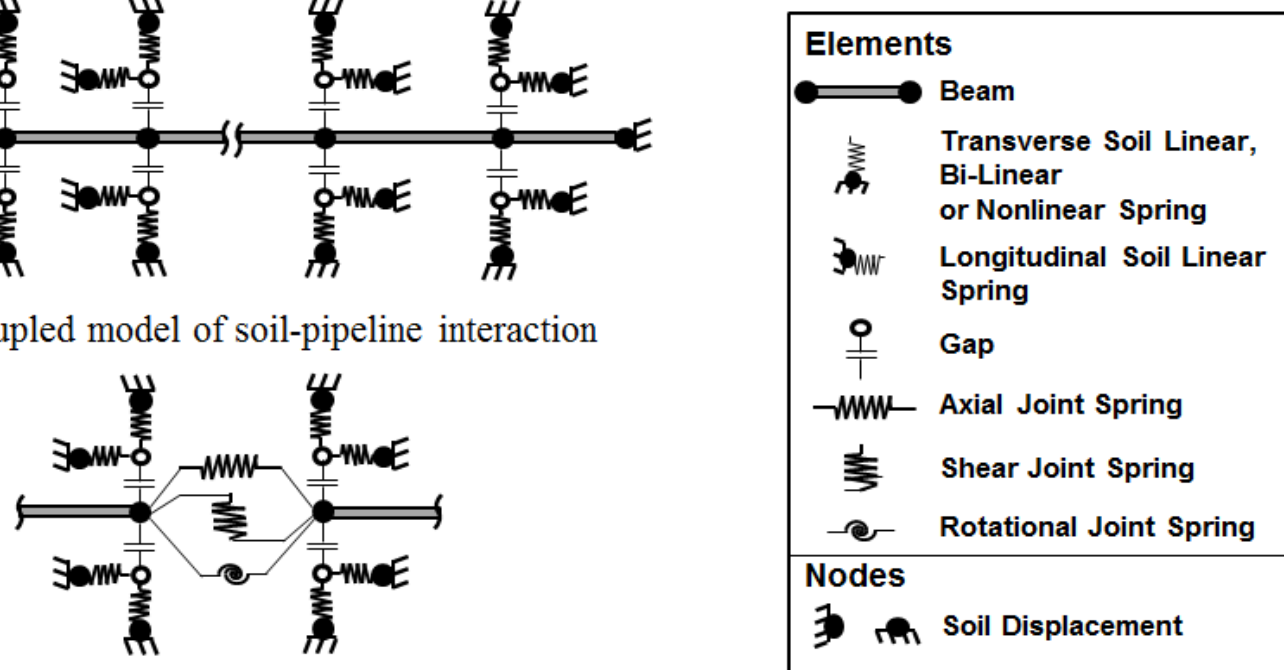

(b) Expanded view for jointed pipeline simulation

Figure 11. 2D FE model setup for coupled forces normal and parallel to a pipeline under PGD 

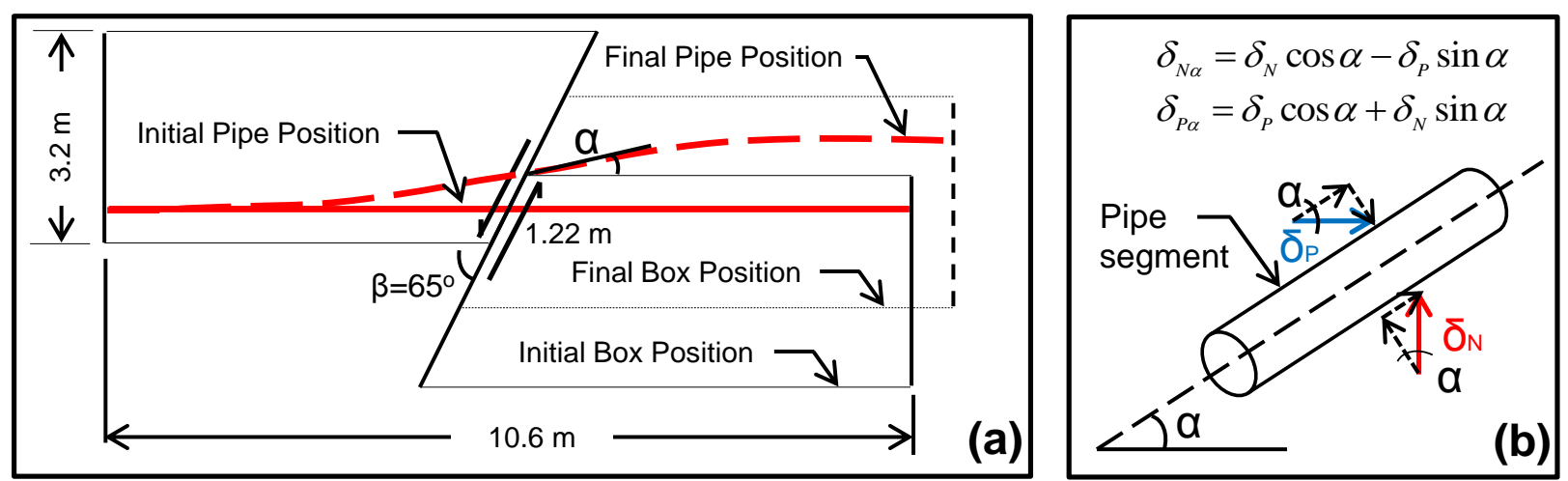

Figure 12. Plan view of large-scale pipeline test showing (a) key dimensions and geometry, and (b) relative pipeline displacements 


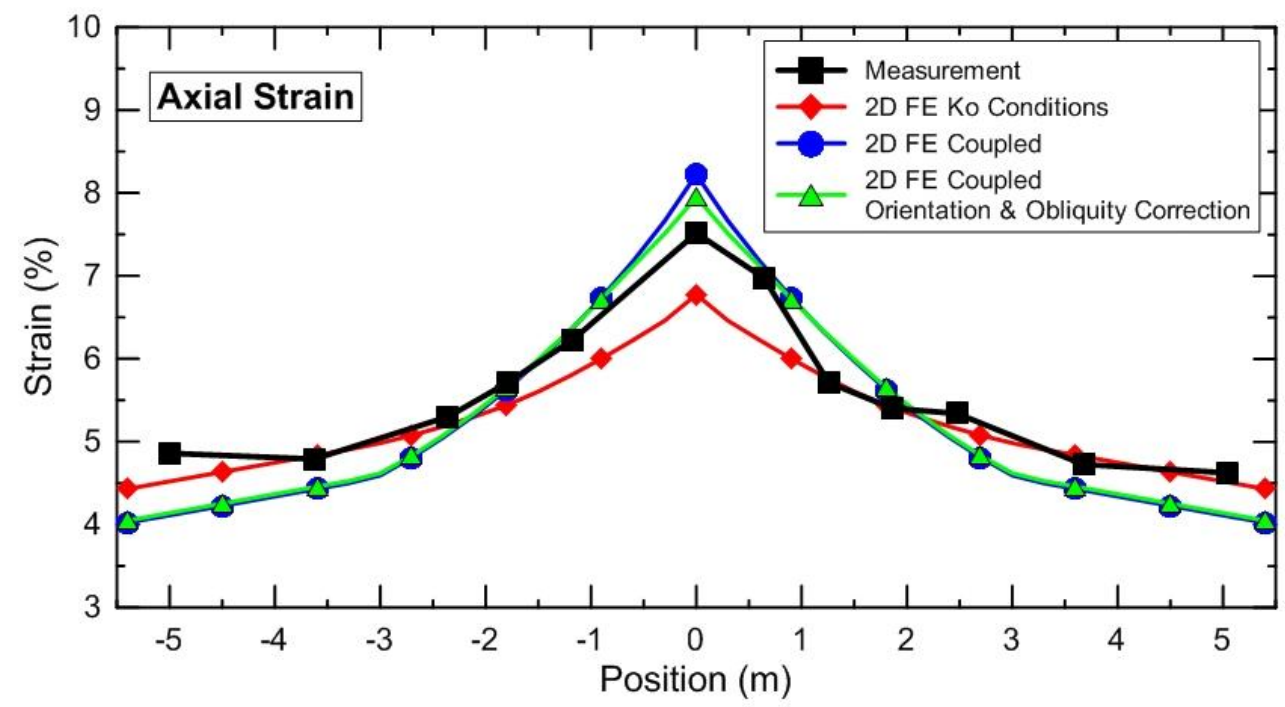

Figure 13. Measured and numerical axial strains in a 250-mm HDPE pipe under strike-slip faulting for various FE models 

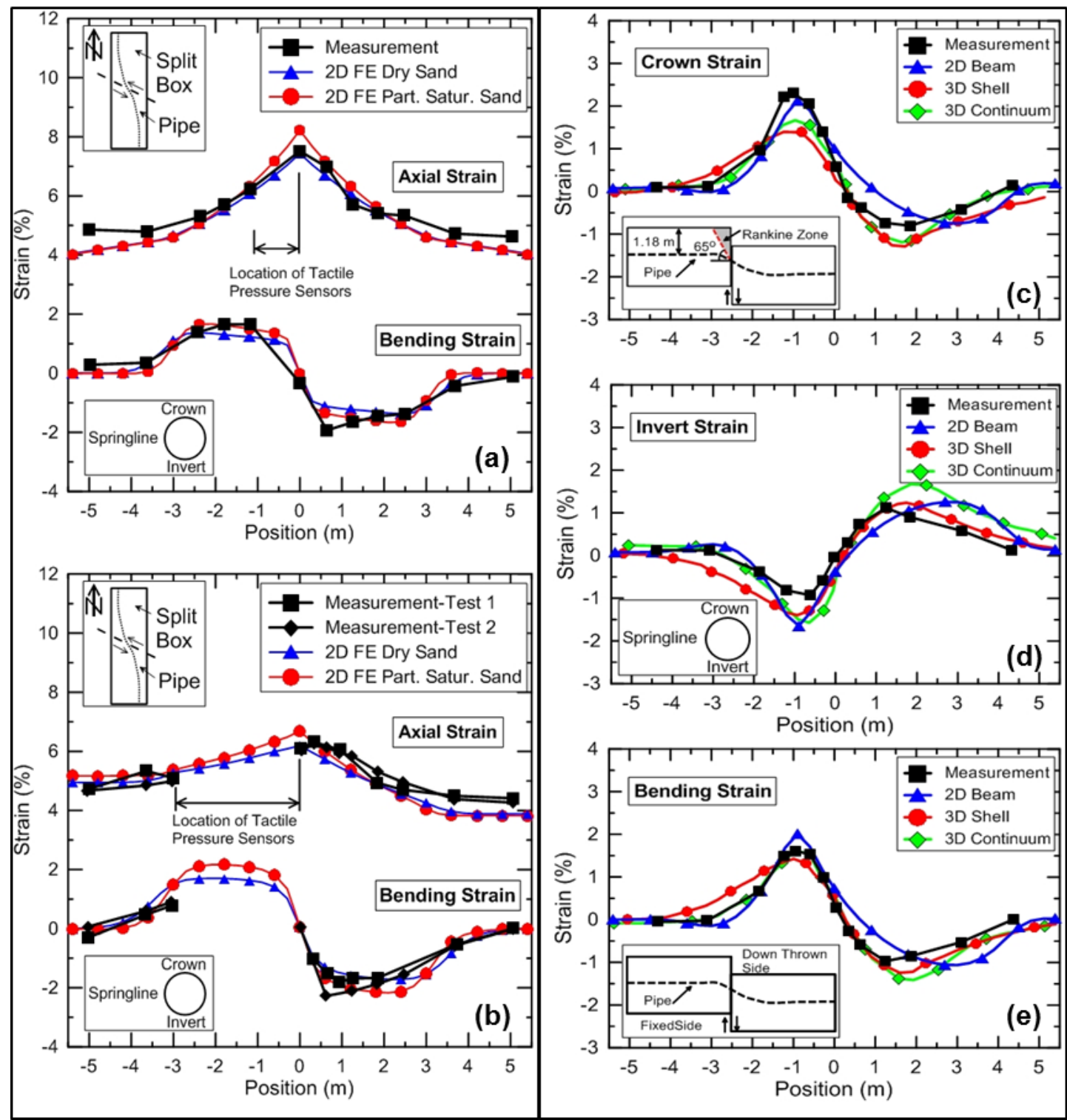

Figure 14. Measured and numerical simulated strains for large-scale testing of (a) 250-mm and (b) 400-mm HDPE pipelines and (c)-(e) centrifuge test of 400-mm HDPE pipeline 